\title{
GLUEBALL AS A BOUND STATE IN THE SELF-DUAL HOMOGENEOUS VACUUM GLUON FIELD *
}

\author{
JA. V. BURDANOV \\ Laboratory of Nuclear Problems, JINR, Russia, \\ E-mail: burdanov@thsun1.jinr.ru \\ G. V. EFIMOV \\ Bogoliubov Laboratory of Theoretical Physics, JINR, Russia \\ E-mail: efimovg@thsun1.jinr.ru
}

\begin{abstract}
Using a simple relativistic QFT model of scalar fields we demonstrate that the analytic confinement (propagator is an entire function in the complex $p^{2}$-plane) and the weak coupling constant lead to the Regge behaviour of the two-particle bound states. In QCD we assume that the gluon vacuum is realized by the selfdual homogeneous classical field which is the solution of the Yang-Mills equations. This assumption leads to analytical confinement of quarks and gluons. We extract the colorless $0^{++}$two-gluon state from the QCD generating functional in the onegluon exchange approximation. The mass of this bound state is defined by the Bethe-Salpeter equation. The glueball mass is $1765 \mathrm{MeV}$ for $\alpha_{s}=0.33$ if the gluon condensate is $\left\langle\left(\alpha_{s} / \pi\right) G G\right\rangle=0.012 \mathrm{GeV}^{4}$.
\end{abstract}

\section{Virton model}

In this section we demonstrate that the analytic confinement and BetheSalpeter (BS) equation in weak coupling regime lead to the pure Regge spectrum for all bound states with radial and orbital quantum numbers.

As an example we use the virton model of scalar field ${ }^{1}$. The equation for the free field $\varphi(x)$ looks like: $\Lambda^{2} e^{\frac{p^{2}}{\Lambda^{2}}} \tilde{\varphi}(p)=0$ and the solution $\varphi(x) \equiv 0$ because the function $e^{\frac{p^{2}}{\Lambda^{2}}} \neq 0$, i.e., it has no zeroes for any real or complex $p^{2}$. Exactly this property means analytic confinement. The field $\varphi(x)$ can exist in the virtual state only, so that it can be called virton field ${ }^{2}$.

The propagator $\Lambda^{2} \tilde{D}\left(p^{2}\right)=e^{-\frac{p^{2}}{\Lambda^{2}}}$ is an entire analytical function in the complex $p^{2}$-plane, i.e., there are no poles corresponding to free particles. It

*This work is supported by RFFI grants $02-02-27064,01-02-17200$ 
guarantees the confinement of "particles" $\varphi(x)$ in each perturbation order over the dimensionless coupling constant $\lambda=\left(\frac{3 g}{4 \pi \Lambda}\right)^{2} \leq 1$. The parameter $\Lambda$ characterizes the scale of the confinement region.

The mass $M_{Q}$ of the bound states with quantum numbers $Q=(n, l)$ is defined by the BS equation in one-boson exchange approximation ${ }^{3}$. The virton model gives the pure Regge behaviour

$$
M_{n l}^{2}=2 \Lambda^{2} \ln \left(\lambda_{c} / \lambda\right)+(l+2 n) \Lambda^{2} \ln \left(2 \lambda_{c}\right) .
$$

We can see that bound states exist for small coupling constant $\lambda<\lambda_{c} \sim 7$ and their masses grow when $\lambda \rightarrow 0$ as $M_{Q} \sim \Lambda \sqrt{2 \ln \left(\lambda_{c} / \lambda\right)}$. The slope of the Regge trajectories is defined by the scale of the confinement region $\Lambda$ and does not depend on the coupling constant $\lambda$.

\section{Glueball mass}

We suppose ${ }^{4}$ that the QCD vacuum is realized by a homogeneous (anti-) self-dual gluon field

$$
B_{\mu}^{a}(x)=n^{a} B b_{\mu \nu} x_{\nu}, \quad b_{\mu \rho} b_{\rho \nu}=-\delta_{\mu \nu}, \quad \tilde{b}_{\mu \nu}=\epsilon_{\mu \nu \alpha \beta} b_{\alpha \beta}= \pm b_{\mu \nu},
$$

which is the solution of the Yang-Mills equations. The parameter $B$ is a tension of the field.

This assumption leads to full quark and partial gluon analytic confinement ${ }^{1,4}$. It means that in the presence of field (1) quark and gluon propagators are entire analytical functions in $p^{2}$ complex plane.

The mechanism of the creation of two-gluon bound states can be described in the following way.

- Gluon-gluon effective Lagrangian in one-gluon exchange approximation is extracted from 3-gluon interaction in Euclidean QCD generating functional in the presence of background field.

- Bilocal colorless scalar gluon currents are derived using the Fierz transformation.

- Untroducing the orthonormal system of functions, bilocal gluon currents can be decomposed into a series of nonlocal scalar colorless currents with radial and orbital quantum numbers.

- Bosonization procedure of the nonlocal 4-gluon interaction consists in a choice of orthonormal functions which correspond to amplitudes of Bethe-Salpeter equation. The kernel of BS equation is written in a symmetric hermitian form that allows one to find the 
ground state BS amplitude using the variational method. The crucial point is that only confined gluons are responsible for arising two-gluon bound states.

- Glueball mass spectrum is determined by BS equation.

The parameter $\Lambda$ can be connected with the gluon vacuum condensate $\left\langle\frac{\alpha_{s}}{\pi} G_{\mu \nu}^{a} G_{\mu \nu}^{a}\right\rangle_{0}=\frac{4}{\pi^{2}}(g B)^{2}=\frac{4}{3 \pi^{2}} \Lambda^{4}$, so that, using the customary value of the gluon condensate ${ }^{5}$ we obtain $\Lambda=546 \mathrm{MeV}$. Our numerical value of scalar glueball mass for ${ }^{6} \alpha_{s}=0.33$ is $M_{0^{++}}=1765 \mathrm{MeV}$. This result is in reasonable agreement with the lattice data ${ }^{7}$.

\section{Conclusion}

In the conclusion we would like to list the main points of our approach.

(i) Analytic confinement and BS equation in a weak coupling regime lead to the Regge spectrum of bound states (see also ${ }^{8}$ ).

(ii) Self-dual homogeneous vacuum gluon field (1) leads to full quark confinement and partial gluon confinement.

(iii) Only confined gluon degrees of freedom are responsible for glueball bound states.

(iv) Numerical result for the mass of the $0^{++}$glueball is quite reasonable and with the results of ${ }^{4}$ gives hope that our main assumption (1) contains "a grain of truth" about the structure of the QCD vacuum.

\section{Acknowledgments}

We wish to thank A. Dorokhov, F. Lenz, S. Mikhailov, S. Nedelko and A. Illarionov for helpful discussions.

\section{References}

1. G.V. Efimov and Ja.V. Burdanov, Phys. Rev. D64, 014001 (2001).

2. G.V. Efimov and M.A. Ivanov, IOP Publishing LTD, London, (1993).

3. G.V. Efimov, hep-ph/9907483 (1999).

4. G.V. Efimov et al, Phys. Rev. D51, 174 (1995), Eur. Phys. J. C1 343 (1998), Phys. Rev. D54 4483 (1996), Phys. Rev. D59 014026 (1999).

5. M.A. Shifman, World Scientific 62, (1999).

6. I. Hinchliffe, Annu. Rev. Nucl. Part. Sci. 50, 643 (2000).

7. C. Morningstar and M. Peardon, Phys. Rev. D60, 034509 (1999).

8. G.V. Efimov and G. Ganbold, Phys. Rev. D65, 054012 (2002). 